\title{
3-Dimensional Cone-Beam Computerized Tomographic Cephalometric Database on Jaw Dimensions in Chinese
}

\author{
Anson C.M. Chau ${ }^{\mathrm{a}}$, Ricky W.K. Wong*,b and Urban Hägg ${ }^{\mathrm{c}}$ \\ ${ }^{a}$ Assistant Professor, Oral Radiology, University of Hong Kong, Hong Kong \\ ${ }^{b}$ Clinical Associate Professor, Orthodontics, University of Hong Kong, Hong Kong \\ ${ }^{c}$ Chair Professor, Orthodontics, University of Hong Kong, Hong Kong
}

\begin{abstract}
Aim: To create the World's first database on 3-dimensional cephalometrics on jaw sizes in Chinese based on cone-beam computerized tomography (CBCT).

Materials and Methods: 40 consecutive CBCT (20 males and 20 females) of a Chinese population of age ranged 21 to 30 were studied and evaluated cephalometric measurements on jaw dimensions.

Results: Six cephalometric measurements Cdl-A, Cdr-A, Cdl-Pg, Cdr-Pg, Cdl-Cdr and A-Pg were measured. All except A-Pg were significantly larger in males.

Conclusion: The availability of a database in a population allows research and clinical use of cephalometrics and comparisons of various craniofacial dimensions among different populations to the 3-dimensional level.
\end{abstract}

Keywords: Cone beam computed tomography, Cone beam, Cephalometry, CBCT, Southern Chinese.

\section{INTRODUCTION}

Knowledge about various dimensions of the upper and lower jaws in a population is important for both anthropological and medical purposes. Computerized tomography (CT) has been used to produce the 3-dimensional (3D) morphology of the skeletal structures of the cranium [1].

In 1998, cone beam computerized tomography (CBCT) systems have been developed specifically for the maxillofacial region [2]. The radiation dose has been suggested to be of similar order of other dental radiographs [3]. In recent years, CBCT applications have included craniofacial assessment in orthodontics and in maxillofacial surgery [4-6]. High dimensional accuracy has been reported for this CBCT in measurement of facial structures [7-10]. The cost-benefits of CBCT scanning are better than the combination of several 2-dimensional (2D) radiographic images with respect to the intrinsic information, and better than CT with respect to radiation dose and cost. Therefore, the replacement of conventional plain radiographs with CBCT appears to be a general trend [11]. Many CBCT devices are capable of a large field of view imaging of the skull to include most anthropometric landmarks used in cephalometric analysis. Cephalometric analyses are used to determine various skeletal and dentoalveolar dimensions and relationships by identifying specific landmarks on both hard and soft tissues to consecutively calculate the spatial and angular dimensions and relationships between them.

*Address correspondence to this author at the $2 / \mathrm{F}$, Orthodontics, Prince Philip Dental Hospital, 34 Hospital Road, Sai Ying Pun, Hong Kong; Tel: 852 28590554; Fax: 852 25593803; E-mail: fyoung@hkucc.hku.hk
Recently, Periago and coworkers [12] compared the accuracy of linear measurements made on CBCT derived 3D surface rendered volumetric images (3D images) to direct measurements made on human skulls. They found while many linear measurements between cephalometric landmarks on 3D images obtained using the Dolphin 3D software generated from $\mathrm{CBCT}$ datasets were statistically significantly different from anatomic dimensions, although most could be considered by the authors to be sufficiently accurate for clinical craniofacial analyses. Our experience with CBCT showed some landmarks that were easy to identify in 2D radiographs were difficult to locate in 3D images in CBCT. Therefore, while it is possible to use CBCT to perform 3D cephalometric analyses, some of the landmarks need to be modified so that they can be located in 3D images with higher validity and reliability.

On the other hand, the success in the use of cephalometrics in a population depends on the availability of a database of the cephalometric data of that population so that the cephalometric norms can be calculated. However, no such a database has ever been published.

The aim of this study is to create the World's first database on 3D cephalometrics on jaw dimensions in Chinese based on CBCT.

\section{MATERIALS AND METHODS}

40 consecutive CBCT (20 males and 20 females) of a Chinese population in Hong Kong SAR, China of age ranged 21 to 30 were studied and evaluated cephalometric measurements on jaw dimensions. Their images were retrieved retrospectively from a database of Oral Radiology Unit of the Prince Philip Dental Hospital. 
For the selection criteria of the subjects, CBCT of subjects having gross craniofacial deformity or asymmetry were excluded from the present investigation. Only CBCT with images including the whole maxilla and whole mandible were included in the study.

Cone beam CT images were acquired using the i-CAT Classic system (Imaging Sciences International Harfield, USA). The patients were positioned according to manufacturer's instructions. The head positions were standardized with the Frankfurt horizontal plane parallel to the floor and the mid-saggital plane perpendicular to the floor. Lateral scout radiographs were made and they were used to check the whole mandible and the whole maxilla were included. Patients were scanned by either one of the protocols described here. The protocols were, a single $360^{\circ}$ rotation, 20second scan, comprising 342 projections (pixel size of $0.4 \mathrm{~mm}$ ) with a $13.0 \mathrm{~cm}$ (diameter) by $13 \mathrm{~cm}$ (height) field of view or a double $360^{\circ}$ rotation, 40 -second scan, comprising 549 projections (pixel size of $0.4 \mathrm{~mm}$ ) with a $15.0 \mathrm{~cm}$ (diameter) by $22 \mathrm{~cm}$ (height) field of view. The protocols were acquired using XoranCat acquisition software (version 3.1.77, Xoran Technologies, Ann Arbor, USA). Exposure parameters were controlled by automatic exposure control.

The CBCT data were exported from the XoranCat software in DICOM multi-file format and imported into SimPlant Pro 12.02 (Materialise N. V., Leuven, Belgium). The program was ran on an IBM-compatible personal computer using MS Windows XP. All constructions and measurements were performed on a 20.1-inch flat panel color active matrix TFT screen (FlexScan S2000, Eizo Nanao Technologies Inc, Cypress, USA) with a resolution of $1600 \times 1200$ at $85 \mathrm{~Hz}$ and a $0.255 \mathrm{~mm}$ dot pitch, operated at 8 bit.
The whole mandible and the anterior part of the maxilla were segmented from other structures in the scan in order to facilitate the measurements in this study. The first step in segmentation was applying thresholds containing Hounsfield Units from 500 to 3071, which represented bone intensity in $\mathrm{CT}$ images in general. Region growing technique was then used to create a mask that contained the mandible and the maxilla within other structures. The mask was edited manually by adjusting the thresholds to ensure that the condyles (usually they have lower Hounsfield Units to the rest of the mandible) of the mandible were included in the mask. A 3D image was generated from the mask.

The measurements were performed on the 3D image. The 3D image was free to rotate in any dimensions in order to verify the positioned landmarks. Landmarks were identified by using a cursor driven by the mouse. The cursor was dragged by holding the left key of the mouse from one landmark to the other in order to provide the distance between them in millimeters (mm). Figs. ( 1 to 4) show the measurements in different orientations of the 3D image of a subject.

By pressing the button 'Print Screen' on the keyboard, the screen image was captured and copied in clipboard and this information was pasted and stored in word-processing files for future reference during landmark relocation.

Standard cephalometric landmarks pogonion $(\mathrm{Pg})$ and point $\mathrm{A}(\mathrm{A})$, lying on the mid-sagittal plane, were used to define the anterior limit of the mandible and maxilla respectively. Pg was taken as the most anterior point on the contour of the chin and A was taken as the innermost (most posterior) point on the contour of the premaxilla between anterior

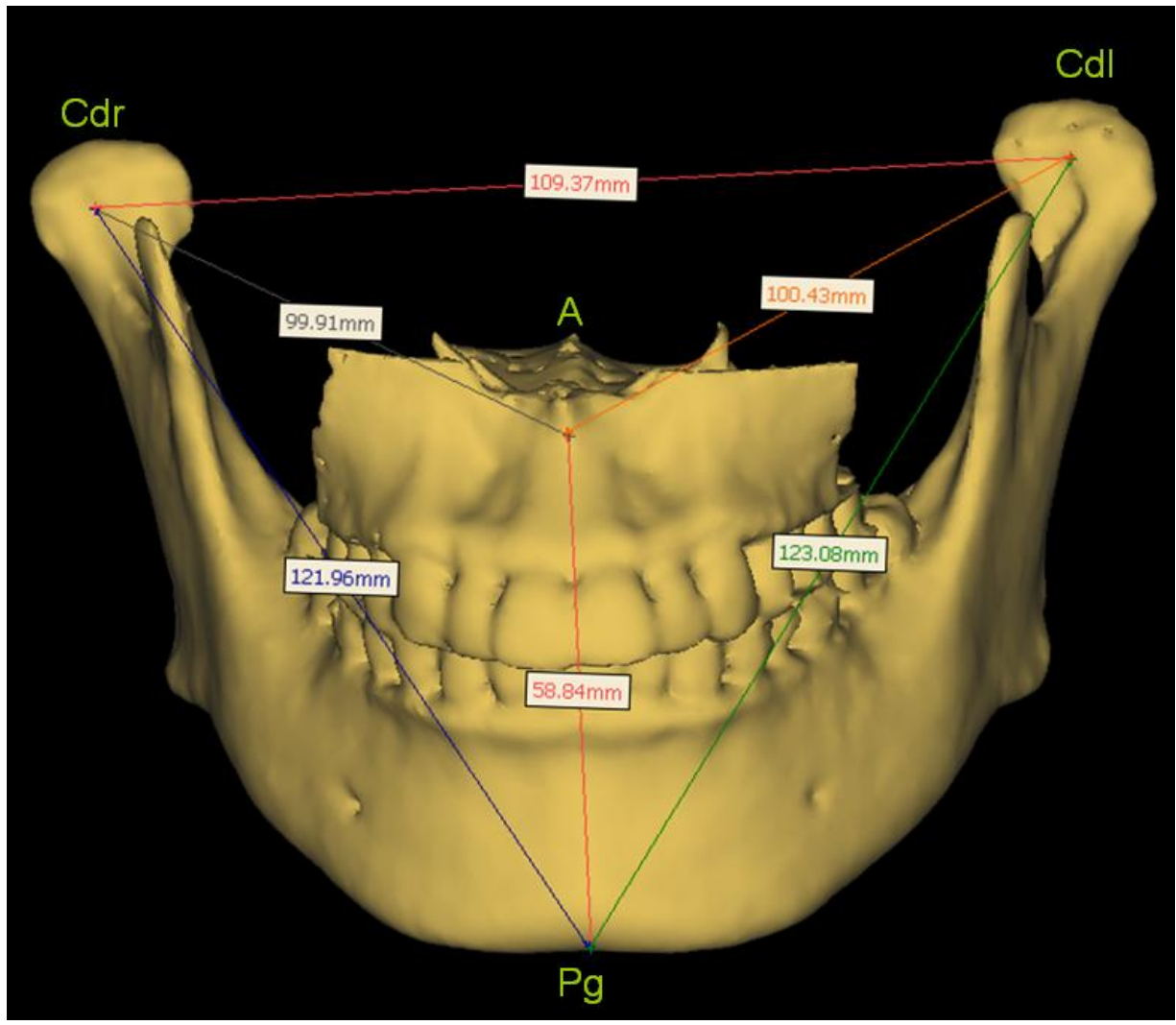

Fig. (1). An antero-posterior view of a 3D model of a subject with measurements. 


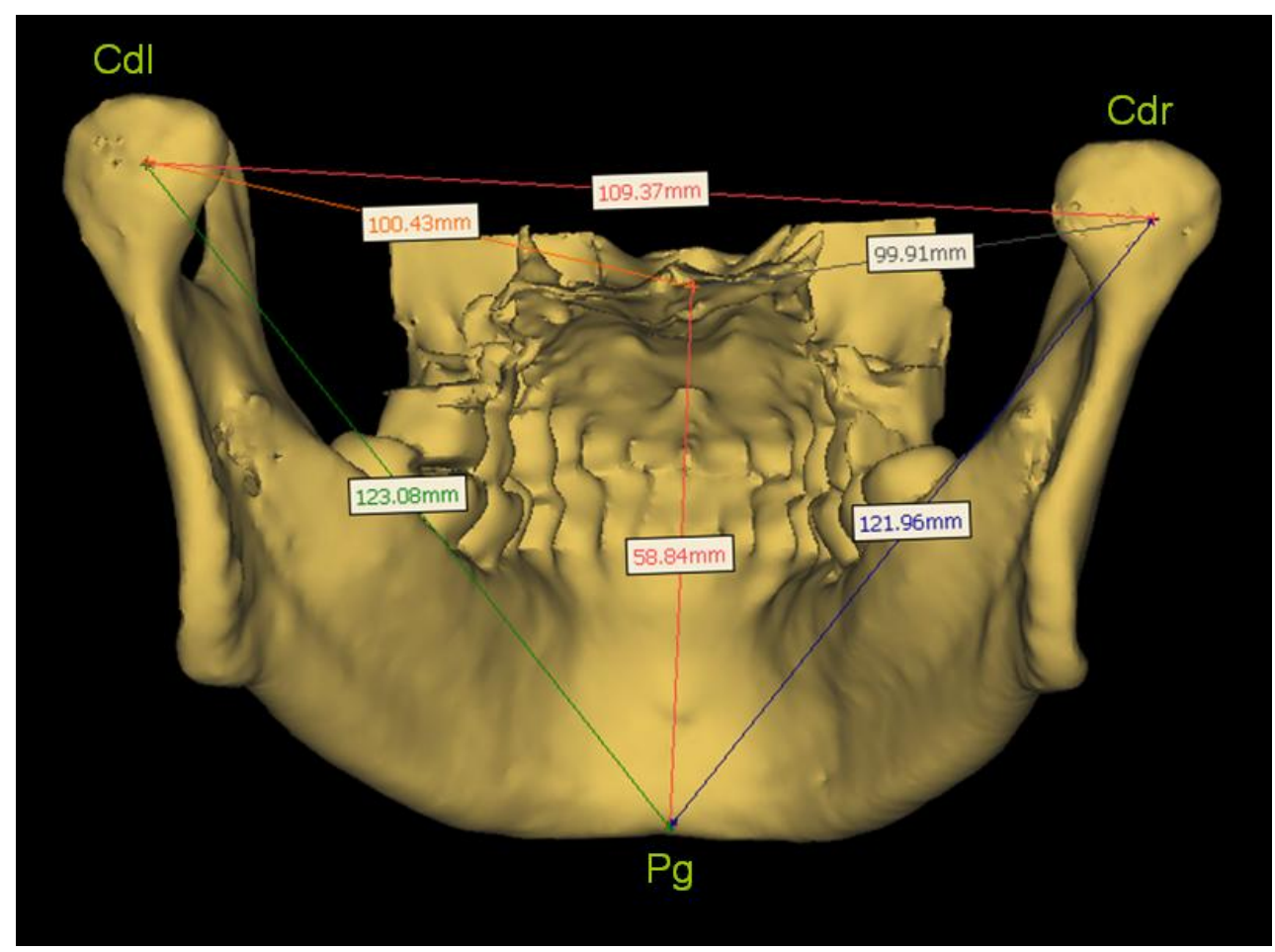

Fig. (2). An postero-anterior view of a 3D model of a subjects with measurements.

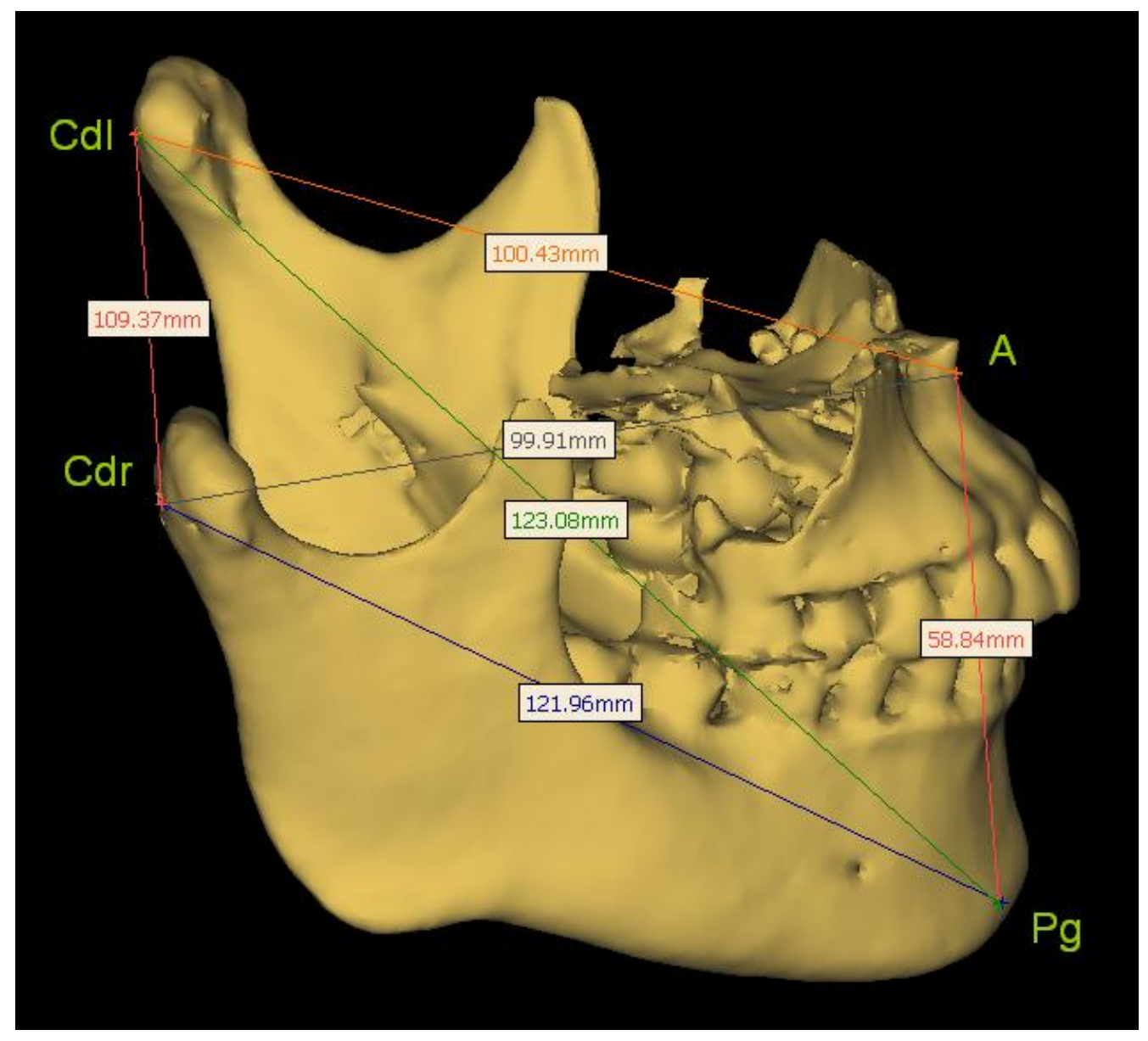

Fig (3). A lateral view of a 3D model of a subject with measurements. 


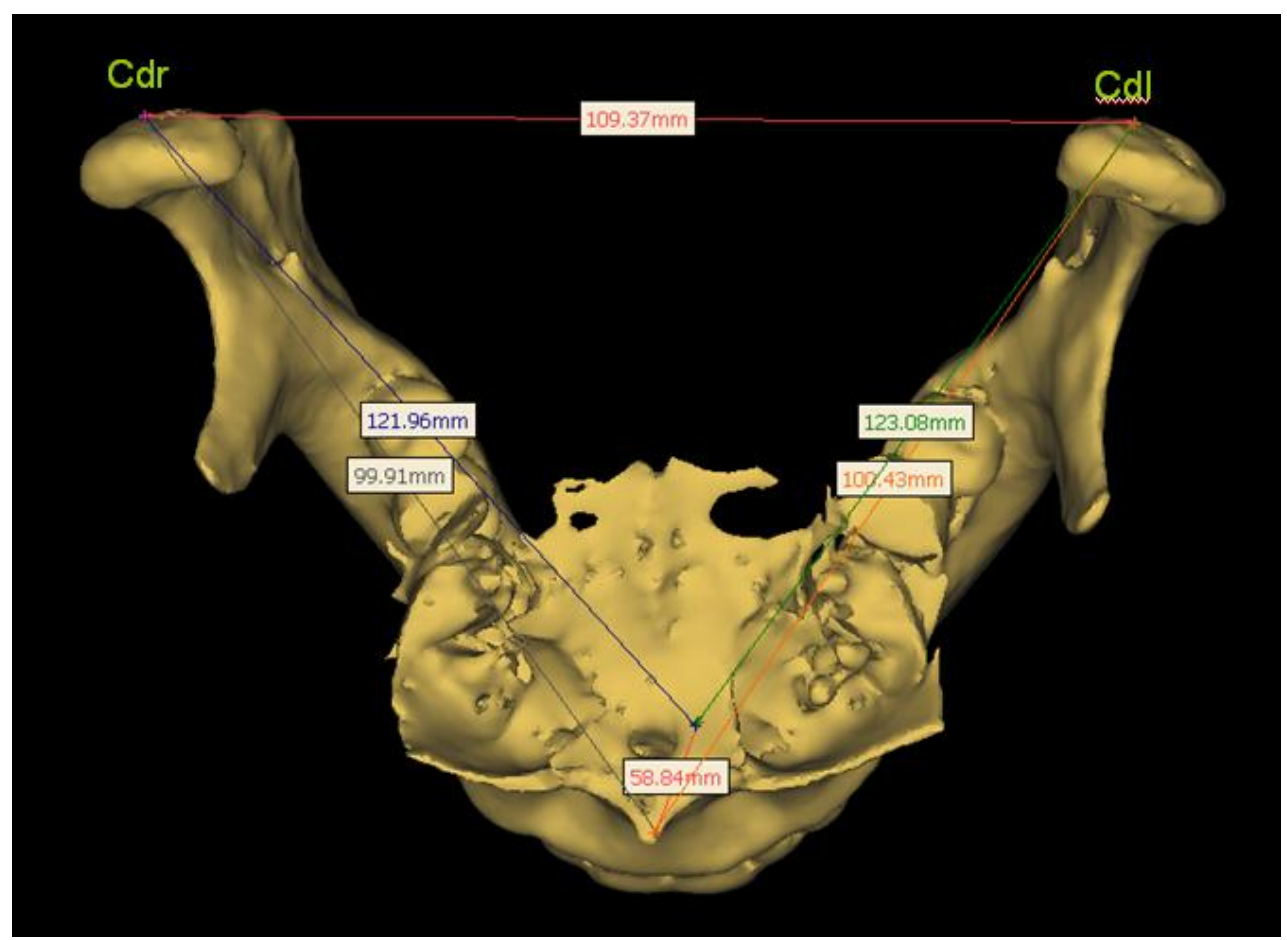

Fig. (4). An supero-inferior view of a 3D model of a subjects with measurements.

nasal spine and the incisor tooth. Modified left and right condylions (Cdl; $\mathrm{Cdr}$ ), which are the most posterior points on the left and right condyles, were used instead of the conventional condylion ( $\mathrm{Co}$ ) because of ease of location by the simultaneous use of 3D images and supplementary tomographic images. These points define the posterior limits of the mandible.

By locating these landmarks, the following dimensions were measured:

$\mathrm{Cdl}-\mathrm{A}, \mathrm{Cdr}-\mathrm{A}$, an indication of the antero - posterior dimensions of the maxilla at left and right sides respectively.

$\mathrm{Cdl}-\mathrm{Pg}, \mathrm{Cdr}-\mathrm{Pg}$, an indication of the antero - posterior dimensions of the mandible at left and right sides respectively.
$\mathrm{Cdl}-\mathrm{Cdr}$, an indication of the transverse dimension of the mandible, and the mid-face.

$\mathrm{A}-\mathrm{Pg}$, an indication of the vertical dimension of the maxilla and mandible, and the anterior lower facial height.

For method error assessment, the cephalometric dimensions of $10 \mathrm{CBCT}$ were measured twice after a time interval of 2 weeks.

Method errors were calculated by Dahlberg's formula [14], M.E $=\sqrt{\frac{\sum d^{2}}{2 n}}$. Where $\sum d^{2}$ is the sum of the squared differences between the two sets of two mean values, and $\mathrm{n}$ is the number of double measurements.

Table 1.

\begin{tabular}{|c|c|c|c|c|c|c|c|c|c|c|c|c|c|c|c|c|}
\hline \multirow[b]{3}{*}{ Variables } & \multicolumn{7}{|c|}{ Males $(n=20)$} & \multicolumn{7}{|c|}{ Females $(n=20)$} & \multirow{3}{*}{\begin{tabular}{c|} 
Sex \\
Difference
\end{tabular}} & \\
\hline & \multirow[b]{2}{*}{ Mean } & \multirow[b]{2}{*}{ SD } & \multirow[b]{2}{*}{ Median } & \multicolumn{2}{|c|}{ Range } & \multicolumn{2}{|c|}{$\begin{array}{c}95 \% \text { Confidence } \\
\text { Interval of the } \\
\text { Mean }\end{array}$} & \multirow[b]{2}{*}{ Mean } & \multirow[b]{2}{*}{ SD } & \multirow[b]{2}{*}{ Median } & \multicolumn{2}{|c|}{ Range } & \multicolumn{2}{|c|}{$\begin{array}{c}95 \% \text { Confi- } \\
\text { dence Interval } \\
\text { of the Mean }\end{array}$} & & \\
\hline & & & & Min & $\operatorname{Max}$ & Lower & Upper & & & & Min & Max & Lower & Upper & & \\
\hline Cdl-A & 101.4 & 5.01 & 101.66 & 87.9 & 109.4 & 99.21 & 103.60 & 95.2 & 4.42 & 94.81 & 89.2 & 106.1 & 93.26 & 97.13 & 6.21 & $* * *$ \\
\hline Cdr-A & 100.8 & 4.38 & 100.83 & 90.7 & 107.7 & 98.90 & 102.70 & 95.9 & 4.26 & 95.45 & 88.5 & 105.6 & 94.05 & 97.77 & 4.90 & $* * *$ \\
\hline Cdl-Pg & 128.9 & 4.18 & 128.88 & 122.1 & 137.0 & 127.06 & 130.73 & 121.7 & 7.03 & 121.89 & 108.0 & 137.7 & 118.62 & 124.78 & 7.19 & $* * *$ \\
\hline Cdr-Pg & 129.0 & 4.76 & 128.55 & 121.7 & 141.0 & 126.89 & 131.05 & 122.8 & 7.70 & 121.89 & 106.2 & 138.2 & 119.40 & 126.16 & 6.19 & $*$ \\
\hline Cdl-Cdr & 103.9 & 4.56 & 103.98 & 96.0 & 113.9 & 101.86 & 105.85 & 102.8 & 6.36 & 102.24 & 92.76 & 117.66 & 100.02 & 105.95 & 1.05 & $*$ \\
\hline A-Pg & 64.2 & 4.57 & 64.25 & 54.7 & 75.7 & 62.17 & 66.18 & 61.3 & 4.11 & 59.99 & 54.6 & 71.2 & 59.47 & 63.07 & 2.91 & \\
\hline
\end{tabular}


Table 2. Method Errors in Six Measurements. Number of Double Measurements is $\mathbf{n}=\mathbf{1 0}$

\begin{tabular}{|c|c|c|c|}
\hline Variables & Measurement 1 & Measurement 2 & Method Errors \\
\hline \hline Cdl-A & 97.6 & 97.4 & 0.03 \\
\hline Cdr-A & 96.9 & 96.7 & 0.06 \\
\hline Cdl-Pg & 124.7 & 123.4 & 0.30 \\
\hline Cdr-Pg & 124.2 & 123.6 & 0.14 \\
\hline Cdl-Cdr & 103.1 & 103.5 & 0.09 \\
\hline A-Pg & 63.5 & 64.8 & 0.31 \\
\hline
\end{tabular}

For statistical analysis, T-test for independent samples was used, and the levels of statistical significance were $P<0.05$

\section{RESULTS}

The cephalometric measurements were summarized in Table 1. There was a large individual variation for all variables for both genders.

Five variables among the Chinese subjects were significantly larger in males: Cdl-A $(\delta: 101.4 \mathrm{~mm}$; $9: 95.2 \mathrm{~mm}$, $\mathrm{P}<0.001)$, Cdr-A ( $\mathrm{O}: 100.8 \mathrm{~mm}$; $+95.9 \mathrm{~mm}, \mathrm{P}<0.01)$, Cdl-Pg (ठ:128.9mm; ᄋ :121.7mm, P<0.01), Cdr-Pg (ठ゚:129.0mm; + :122.8mm, $\mathrm{P}<0.01), \mathrm{Cdl}-\mathrm{Cdr}\left(\delta^{1}: 103.9 \mathrm{~mm}\right.$; $+102.8 \mathrm{~mm}$, $\mathrm{P}<0.05)$. Variable A-Pg $\left(\delta^{\lambda}: 64.2 \mathrm{~mm} ;\right.$ + $\left.: 61.3 \mathrm{~mm}\right)$ had no significant differences between males and females in this study.

The method errors did not exceed $0.5 \mathrm{~mm}$ for any variables Table 2 .

\section{DISCUSSION}

The introduction of CBCT allows possibility to generate 3D volumetric renderings easily using third-party personal computer-based software, making it possible to identify craniofacial structures more naturally. This will undoubtedly expand the use and application of 3D imaging, particularly in the field of orthodontics and maxillofacial surgery [15-18]. However, landmark identification in $3 \mathrm{D}$ is not simple. Up to now no standards have been proposed or accepted for 3D landmarks and the possibility of using anatomic features [19, 20]. One reason for the difficulty in locating cephalometric landmarks on the $3 \mathrm{D}$ images derived from CBCT is that there is a lack of 'depth' perception in these images, in other words, they are not stereoscopic. Therefore, points that can be easily located on dried skulls or 2D radiographs, e.g. condylion, gnathion, can be very difficult to locate on these $3 \mathrm{D}$ images with a high degree of accuracy. To alleviate this problem, it is necessary to use the supplementary tomographic images in vertical, horizontal and sagittal planes to help locating the points on the 3D images. This is made possible by using the points at the limits on various planes, e.g. most anterior, most posterior, most superior, most inferior, most left and most right. Therefore, modified left and right condylions (Cdl; $\mathrm{Cdr})$, which are the most posterior points on the left and right condyles, were used instead of the conventional condylion (Co); pogonion (Pg) was used instead on gnathion $(\mathrm{Gn})$ in this study for construction of the database.

The success in application of workable cephalometric landmarks in 3D images allowed the possibility of creation of the World's first database on 3D cephalometrics on Chinese, which is one-forth of the World's population. At this stage only data on jaw dimensions are measured. Work is in progress to extend the database to other dimensions. The availability of a database in a population allows the clinical use of cephalometrics to 3D level, thus increase the accuracy in diagnosis and treatment. It also allows comparisons of various craniofacial dimensions among different populations to $3 \mathrm{D}$ level, promoting research and advancing the understanding in anthropology.

This age group of Chinese was chosen for measurement because the jaw growth is completed and the effect of aging is minimal. Further studies are needed to include the measurements of other age groups into the database. The subjects of this study were patients coming for dental treatments like removal of impacted teeth. Care was taken to exclude patients with craniofacial deformities and skeletal asymmetries to avoid deviation of the results. Attempts were made to screen the normal population with no skeletal deformity assessed with surgical and orthodontic criteria. Ideally, the sample should be randomized with the general population. However, a study of this type with random sample is virtually impossible because CBCT produces radiation exposure, it does not fulfill the ethical requirements. A retrospective study of the CBCT records with consecutive subjects is the only possible option. Further study with an enlarged sample size is needed to improve validity.

The diet for this Chinese population is similar to that of the Southeast Asia and the staple diet is refined rice. This is different from the Northern Chinese population where the major carbohydrate intake is wheat. Further study is needed to investigate the association between the diet and craniofacial size and morphology.

\section{CONCLUSION}

The availability of a database in a population allows research and clinical use of cephalometrics and comparisons of various craniofacial dimensions among different populations to the 3-dimensional level. 


\section{REFERENCES}

[1] Hounsfield GN. Computerized transverse axial scanning (tomography). 1. Description of system. Br J Radiol 1973; 46: 1016-22.

[2] Mozzo P, Procacci C, Tacconi A, Martini P T, Andreis I A . A new volumetric CT machine for dental imaging based on the conebeam technique: preliminary results. Eur Radiol 1998; 8: 558-64.

[3] Ludlow J B, Davies-Ludlow L E, Brooks S L, Howerton WB. Dosimetry of 3 CBCT devices for oral and maxillofacial radiology: CB Mercuray, NewTom $3 \mathrm{G}$ and i-CAT. Dentomaxillofac Radio 2006; 35: 219-26.

[4] Maki K, Inou N, Takanishi A, Miller A J. Computer-assisted simulations in orthodontic diagnosis and the application of a new cone beam X-ray computed tomography. Orthod Craniofac Res 2003; 6(suppl 1): 95-101.

[5] Aboudara C A, Hatcher D, Nielsen I L, Miller A. A threedimensional evaluation of the upper airway in adolescents. Orthod Craniofac Res 2003; 6(Suppl 1): 173-75.

[6] Danforth R A, Dus I, Mah J. 3-D volume imaging for dentistry: a new dimension. J Calif Dent Assoc 2003; 31: 817-23.

[7] Kobayashi K, Shimoda S, Nakagawa Y, Yamamoto A. Accuracy in measurement of distance using limited cone-beam computerized tomography. Int J Oral Maxillofac Implants 2004; 19: 228-31.

[8] Lascala C A, Panella J, Marques M M. Analysis of the accuracy of linear measurements obtained by cone beam computed tomography (CBCT-NewTom). Dentomaxillofac Radiol 2004; 33: 291-4.

[9] Beason R, Brooks SL. TMJ imaging accuracy using alpha prototype of DentoCAT_cone-beam CT [abstract]. J Dent Res 2004; 83(special issue A) 1938.

[10] Hilgers ML, Scarfe WC, Scheetz JP, Farman AG. Accuracy of linear TMJ measurements with cone beam computed tomography and digital cephalometric radiography. Am J Orthod Dentofacial Orthop 2005; 1278: 803-11.
[11] Mah J, Hatcher DC. Craniofacial imaging in orthodontics. In: Graber TM, Vanarsdall RL, Vig KWL, Eds. Orthodontics: current principles and techniques. St Louis: Elsevier 2005; pp. 71-100.

[12] Periagoa DR, Scarfeb WC, Moshiric M, Scheetzd JP, Silveirae AM, Farmanf AG. Linear accuracy and reliability of cone beam CT derived 3-dimensional images constructed using an orthodontic volumetric rendering program. Angle Orthod 2008; 78: 387-95.

[13] Karlberg P, Taranger J. The Somatic Development of Children in a Swedish Urban Community. Acta Paediatr Scand 1976; (Suppl 258): 7-76

[14] Dahlberg, G. Statistical methods for medical and biological students. Allen \& Unwin, London 1940: p. 126.

[15] Danforth R A, Dus I, Mah J. 3-D volume imaging for dentistry: a new dimension. J Calif Dent Assoc 2003; 31: 817-23.

[16] Kau C H, Richmond S, Palomo J M, Hans M G. Three-dimensional cone beam computerized tomography in orthodontics. J Orthod 2005; 32: 282-93.

[17] Cevidanes L H, Styner M A, Proffit W R. Image analysis and superimposition of 3-dimensional cone-beam computed tomography models. Am J Orthod Dentofac Orthop 2006; 129: 611-8.

[18] Weinberg S M, Kolar J C. Three-dimensional surface imaging: limitations and considerations from the anthropometric perspective. J Craniofac Surg 2005; 16: 847-51.

[19] Lagravere M O, Major P W. Proposed reference point for 3dimensional cephalometric analysis with cone-beam computerized tomography. Am J Orthod Dentofacial Orthop 2005; 128: 657-60.

[20] Lou L, Lagravere M O, Compton S, Major P W, Flores-Mir C. Accuracy of measurements and reliability of landmark identification with computed tomography $(\mathrm{CT})$ techniques in the maxillofacial area: a systematic review. Oral Surg Oral Med Oral Pathol Oral Radiol Endod 2007; 104: 402-11.

(C) Chau et al.; Licensee Bentham Open.

This is an open access article licensed under the terms of the Creative Commons Attribution Non-Commercial License (http://creativecommons.org/licenses/by-nc/3.0/) which permits unrestricted, non-commercial use, distribution and reproduction in any medium, provided the work is properly cited. 